\title{
ORIGINAL ARTICLE \\ The use of bronchodilators in people with recently acquired tetraplegia: a randomised cross-over trial
}

\author{
DJ Barratt ${ }^{1,2}$, LA Harvey ${ }^{1}$, PA Cistullii, ${ }^{2,3}$ L Nier $^{2}$ and S Denis ${ }^{4}$
}

\begin{abstract}
Study design: A within-participant, double-blind, cross-over, randomised control trial. Objectives: To determine the short-term effects of bronchodilator therapy on respiratory function in people with recently acquired motor complete tetraplegia.

Setting: Hospital, Australia.

Methods: A total of 12 people with recently acquired tetraplegia were randomised to receive either a one-off dose of a bronchodilator followed by an equivalent dose of a placebo propellant between 1 day and 1 week later or visa versa. The three outcomes were forced expiratory volume in $1 \mathrm{~s}$ (FEV1), peak expiratory flow rate (PEF) and forced vital capacity (FVC). These were measured while supine by a blinded assessor 10 and 30 min after treatment. Data were analysed on 11 participants and reported as percentage of predicted. Results: The FEV1, FVC and PEF mean between-group differences (95\% confidence interval) at 10 min post treatment were $7.3 \%$ $(2.7-11.9 \% ; P=0.003), 5.5 \%(1.6-9.4 \% ; P=0.008)$ and $20.1 \%(1.1-40.4 \% ; P=0.039)$. Similar effects were observed at 30 min for FVC and FEV1 but not for PEF.

Conclusion: Bronchodilator therapy has a beneficial effect on FEV1, FVC and PEF in participants with recently acquired tetraplegia. Spinal Cord (2012) 50, 836-839; doi:10.1038/sc.2012.62; published online 29 May 2012
\end{abstract}

Keywords: spinal cord injuries; tetraplegia; spirometry; bronchodilator; respiratory

\section{INTRODUCTION}

People with tetraplegia are highly susceptible to respiratory complications in the early days after injury. This is due to a number of factors including the adverse effects of respiratory muscle paralysis, imposed immobility and pain. ${ }^{1}$ Recent clinical guidelines have advocated the use of bronchodilator therapy as an effective way to treat and prevent respiratory complications. ${ }^{2}$ However, these recommendations are not based on clinical trials involving patients with recently acquired tetraplegia. Instead, they are based on trials involving people with established tetraplegia. Trials in people with established tetraplegia have demonstrated that bronchodilator therapy improves forced expiratory volume in $1 \mathrm{~s}\left(\mathrm{FEV}_{1}\right)$ and peak expiratory flow rate (PEF) and aids the removal of secretions. ${ }^{3-7}$ However, it cannot be assumed that the response of people with recently acquired tetraplegia to bronchodilator therapy is the same as the response of people with established tetraplegia. One important difference may be the implications of the isolated sympathetic nervous system. Although all patients with complete tetraplegia loose supraspinal control of the sympathetic nervous system, those recently injured may also have an interim loss of reflex-mediated sympathetic activity associated with spinal shock. This may last for $1-3$ months. ${ }^{8}$ It is not clear how loss of reflex-mediated sympathetic activity in the first few months after injury affects a patient's response to bronchodilator therapy. The purpose therefore of this study was to determine the immediate effects of bronchodilator therapy on respiratory function in people with recently acquired tetraplegia.

\section{MATERIALS AND METHODS \\ Participants}

A within-participant, double-blind, cross-over randomised control trial was undertaken where participants received both treatment and placebo interventions between 1 day and 1 week of each other. All the patients admitted to a Sydney spinal injury unit were screened for inclusion between May 2009 and February 2012. Inclusion was based on a recent ( $<8$ weeks) motor complete cervical SCI (that is, an American Injury Impairment Scale (AIS) A or B according to the International Standards for Neurological Classifications of Spinal Cord Injury). Exclusion criteria were pre-existing diagnosis of asthma, other diagnosed respiratory diseases (chronic obstructive pulmonary disease and emphysema), longterm pre-morbid bronchodilator-therapy use or unstable respiratory status at the time of screening. Smokers and mild undiagnosed asthmatics were not excluded.

All the participants were randomised to receive either the placebo or the bronchodilator-therapy treatment first. They received the alternate intervention within a median (interquartile range) of 6 days (4-7) later. Randomisation occurred after baseline measurements to ensure participants were able to perform acceptable spirometry according to the American Thoracic Society guidelines modified for SCI. ${ }^{9}$ The randomisation schedule was computer generated by an independent person and stored off site in opaque sequentially numbered envelopes. Once an envelope was opened, the participant was considered to have entered the trial.

${ }^{1}$ Rehabilitation Studies Unit, Northern Clinical School, Sydney Medical School, University of Sydney, Sydney, New South Wales, Australia; ${ }^{2}$ Spinal Cord Injury Unit, Royal North Shore Hospital, Sydney, New South Wales, Australia; ${ }^{3}$ Department of Respiratory Medicine, Royal North Shore Hospital, University of Sydney, Sydney, New South Wales, Australia and ${ }^{4}$ Department of Physiotherapy, Prince of Wales Hospital, Sydney, New South Wales, Australia

Correspondence: Associate Professor LA Harvey, Rehabilitation Studies Unit, Northern Clinical School, Sydney Medical School, University of Sydney, PO Box 6, Ryde, Sydney, New South Wales 1680, Australia.

E-mail: I.harvey@usyd.edu.au

Received 23 February 2012; revised 17 April 2012; accepted 27 April 2012; published online 29 May 2012 
Both the placebo and bronchodilator therapy (Inhaler, salbutamol (as sulfate) $100 \mu \mathrm{g}$ per actuation) were administered with a puffer via a spacer. After four puffs were dispensed into the spacer chamber, the participant was instructed to perform deep breaths with inspiratory holds of $3 \mathrm{~s}$ for a period of $1 \mathrm{~min}$. This was performed while supine. Participants remained supine until all assessments were completed. Therapist and participant blinding were achieved by ensuring that an independent person prepared each placebo or bronchodilator therapy solution according to the randomisation schedule. Puffers were taped to disguise the contents. The placebo solution contained propellant only. All outcome measures were taken by a blinded assessor. Three outcomes were measured, namely, forced vital capacity (FVC), FEV1 and PEF. These were performed while supine using a calibrated spirometer (spirolab II, manufactured by MIR, Rome, Italy) 10 and 30 min after inhalation of spacer contents. Three FVC manoeuvres were performed but only the highest results were recorded. The original protocol specified $1 \mathrm{~h}$ follow-up measurements of all the outcomes but this was abandoned because participants were unwilling to lie flat for an hour.

\section{Statistical analysis}

Initially, one-way ANOVAs were used to test for period effects. That is, effects solely due to the passage of time. None were detected $(P<0.05)$ and hence data were analyzed using a two-way ANOVA to determine the interaction between intervention (that is, bronchodilator therapy versus placebo) and assessment (that is, pre versus post versus follow-up). Data on one participant who was clearly uncooperative in the second round of testing was excluded from the analysis.

\section{RESULTS}

The flow of participants through the study is shown in Figure 1. A total of 118 patients admitted with AIS A and AIS B tetraplegia were screened for inclusion into the study and 12 were ultimately randomised. One participant was a smoker but he was the uncooperative patient whose data were excluded from the analyses. Participants received both interventions according to the protocol, and all the participants and the assessor remained blind for all aspects of the study. The median (interquartile range) age and time since injury were 30 (25-37) years and 24 (18-35) days, respectively. All the participants had motor complete lesions with neurological levels ranging from C5 to C7 (Table 1). Ten participants had AIS A lesions and two participants had AIS B lesions.

Table 2 summarises the results. The FEV1, FVC and PEF mean between-group differences ( $95 \%$ confidence interval) at $10 \mathrm{~min}$ post treatment were $7.3 \%(2.7-11.9 \% ; P=0.003), 5.5 \% \quad(1.6-9.4 \%$; $P=0.008)$ and $20.1 \%(1.1-40.4 \% ; P=0.039)$. Similar effects were observed at $30 \mathrm{~min}$ for FVC and FEV1 but not for PEF.

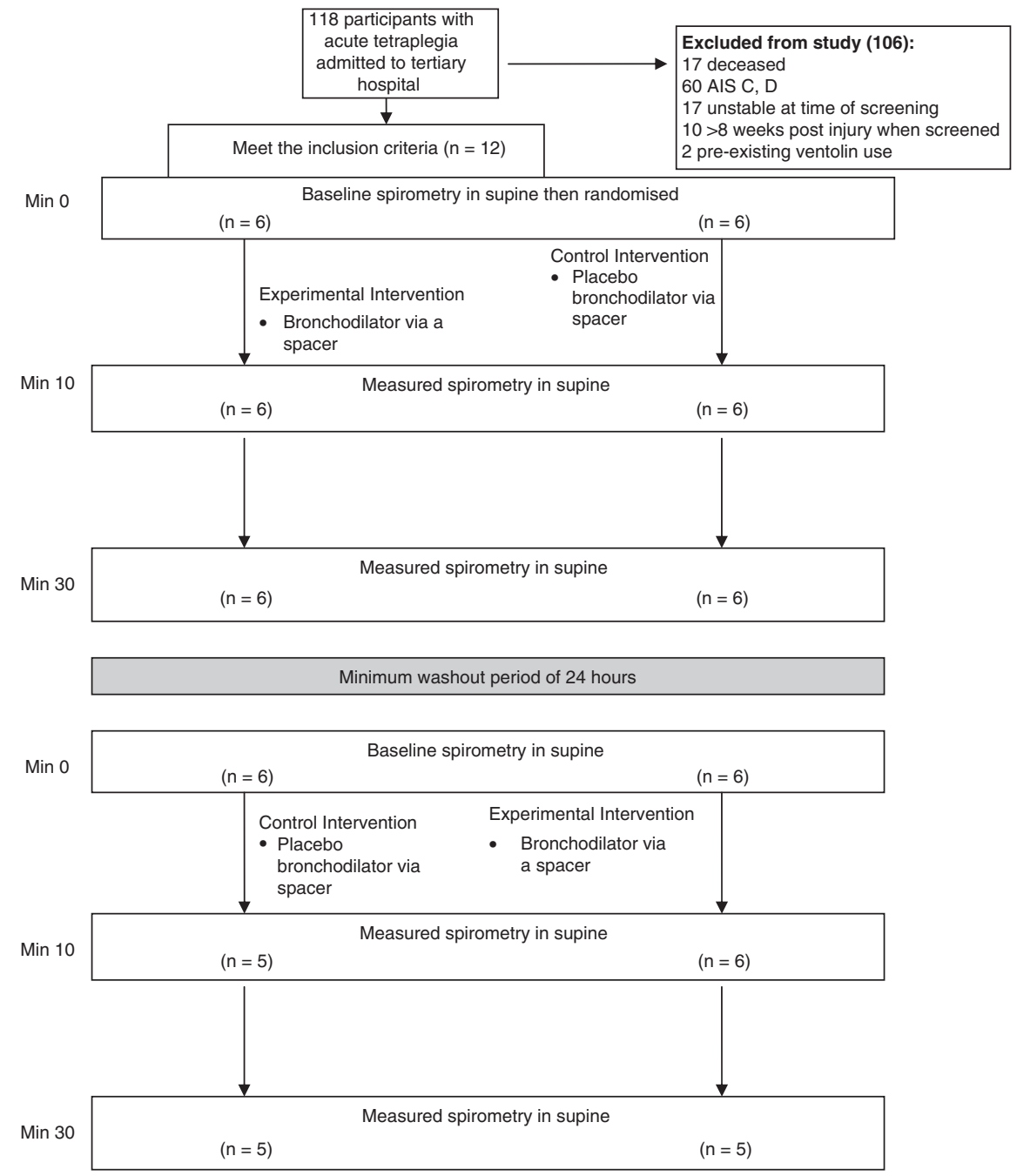

Figure 1 Flow of participants through the trial. 


\section{DISCUSSION}

This is the first randomised controlled trial to investigate the shortterm effects of bronchodilator therapy on respiratory function in people with recently acquired tetraplegia. It is important to determine the effectiveness of bronchodilator therapy because this therapy is widely advocated but its efficacy has only been investigated in people with established tetraplegia. People with recently acquired tetraplegia may respond differently to bronchodilator therapy than people with established tetraplegia because of their interim loss of sympatheticmediated reflexes immediately after injury. The results indicate a small and statistically significant immediate effect of bronchodilator therapy on $\mathrm{FEV}_{1}$, FVC and PEF that was sustained at $30 \mathrm{~min}$ for $\mathrm{FEV}_{1}$ and FVC.

The improvement in $\mathrm{FEV}_{1}$ with bronchodilator therapy may be indicative of underlying bronchoconstriction in people with recently acquired tetraplegia. It is not surprising that PEF in this group did not improve. Peak flow in this group is primarily limited by expiratory muscle weakness. Any changes in airway diameter due to bronchodilator therapy would not influence the underlying weakness. Improvements in FEV1 without any change in expiratory muscle strength indicate a decrease in airway resistance that can be attributed to smooth muscle relaxation. Bronchoconstriction has been reported in people with established tetraplegia but not examined in people with recently acquired tetraplegia. ${ }^{3-7}$ The physiological basis for bronchoconstriction in people with established tetraplegia is debated,

Table 1 Characteristics of participants including age (years), time since injury (days), gender, AIS classification and neurological level

\begin{tabular}{ll}
\hline & Participants $\mathrm{n}=11$ \\
\hline Age (years), median (IQR) & $30(25-37)$ \\
Time (days) since injury, median (IQR) & $24(18-35)$ \\
Male:female participants, $n$ & $9: 3$ \\
AIS A:B, $n$ & $10: 2$ \\
& \\
Motor level, $n$, & \\
C5 & 8 \\
C6 & 2 \\
C7 & 1 \\
PEF(I min & \\
FVC(I) & \\
FEV1(I) & $3.76(1.3)$ \\
\hline Abbriations: AIS, An & $2.29(0.5)$ \\
\end{tabular}

Abbreviations: AIS, American Injury Impairment Scale; FEV1, forced expiratory volume in $1 \mathrm{~s}$; FVC, forced vital capacity; IQR, interquartile range; PEF, peak expiratory flow rate. although it is most commonly attributed to loss of supraspinal control of the sympathetic nervous system. ${ }^{8}$ The sympathetic nervous system is important for dampening parasympathetic nervous system activity. Without its dampening effects, the parasympathetic nervous system can stimulate the bronchial smooth muscles causing bronchoconstriction. ${ }^{10}$ Bronchoconstriction may be more prominent in people with recently acquired tetraplegia than those with established tetraplegia because of the effects of spinal shock. Spinal shock results in an interim loss of reflexes. The loss of the sympathetic reflexes may result in unmodulated parasympathetic nervous system activity, thereby precipitating bronchoconstriction. It is also likely that bronchoconstriction in this population occurs as a result of breathing at low lung volumes. ${ }^{11-14}$ Perhaps a more important feature of spinal shock in this population is reduced reflex-mediated sympathetic input to the adrenal gland. ${ }^{15}$ This results in a low level of circulating catecholamines and is responsible for the haemodynamic instability observed in acute tetraplegia. Circulating catecholamines from the adrenal gland directly stimulate the B2 adrenoreceptors in lungs, causing bronchodilation of smooth muscles. ${ }^{16}$ Without circulating catecholamines during spinal shock, there is less stimulus for bronchodilation resulting in bronchoconstriction.

Some participants did not respond as well to the bronchodilator therapy as others. One possible explanation is the differences in underlying bronchoconstriction. This may have been due to participants' varying levels of spinal shock. We crudely defined spinal shock by the absence of the patella tendon reflex, although this definition is debated. Only three participants had an absent patella tendon reflex, suggesting that most were not in spinal shock, yet the median time since injury was 24 (18-35) days, suggesting that most were in spinal shock. Regardless, the results reflect the response of people with recent tetraplegia. Responses may also have been influenced by the participants' levels of co-operation. Spirometry is effort-dependant and influenced by factors such as mood and fatigue.

Some of the noted between-group differences may have in part been due to a decline in FEV1 and FVC in response to the placebo rather than an increase in FEV1 and FVC in response to the bronchodilator therapy. The propellant in the placebo solution may have acted as an irritant indicative of airway hyperactivity. Airway hyperactivity has been previously demonstrated in participants with established tetraplegia in response to methacholine and capsin and is also an important feature of asthma. ${ }^{2,4}$ Future studies could clarify this issue by including a third arm of the study in which participants received no intervention.

The effect of bronchodilator therapy on all the outcomes was moderate. The mean effect on FEV1 was $7.3 \%$ of predicted but there

Table 2 Mean (s.d.) 10 and 30 min pre and post FEV1, PEF and FVC for bronchodilator therapy and placebo interventions (percentage of predicted). The mean $(95 \% \mathrm{Cl})$ between-group differences are also shown

\begin{tabular}{|c|c|c|c|c|c|c|c|c|c|c|c|c|}
\hline \multirow[t]{3}{*}{ Outcome } & \multicolumn{6}{|c|}{ Groups } & \multicolumn{4}{|c|}{ Difference within groups } & \multicolumn{2}{|c|}{ Difference between groups } \\
\hline & \multicolumn{2}{|c|}{ Baseline } & \multicolumn{2}{|c|}{$10 \mathrm{~min}$} & \multicolumn{2}{|c|}{$30 \mathrm{~min}$} & \multicolumn{2}{|c|}{$10 \mathrm{~min}$-baseline } & \multicolumn{2}{|c|}{$30 \mathrm{~min}$-baseline } & \multirow{2}{*}{$\frac{10 \mathrm{~min} \text {-baseline }}{\text { Exp-Con }}$} & \multirow{2}{*}{$\frac{30 \mathrm{~min} \text { - baseline }}{\text { Exp-Con }}$} \\
\hline & $\begin{array}{c}\operatorname{Exp} \\
(\mathrm{n}=11)\end{array}$ & $\begin{array}{c}\text { Con } \\
(\mathrm{n}=11)\end{array}$ & $\begin{array}{c}\text { Exp } \\
(\mathrm{n}=11)\end{array}$ & $\begin{array}{c}\text { Con } \\
(\mathrm{n}=11)\end{array}$ & $\begin{array}{c}\operatorname{Exp} \\
(\mathrm{n}=11)\end{array}$ & $\begin{array}{c}\text { Con } \\
(\mathrm{n}=11)\end{array}$ & Exp & Con & Exp & Con & & \\
\hline $\begin{array}{l}\left.\text { PEF(I } \min ^{-1}\right) \\
\text { FVC(I) } \\
\text { FEV1(I) }\end{array}$ & $\begin{array}{l}42(13.3) \\
49(9.6) \\
46(11.1)\end{array}$ & $\begin{array}{l}45(15.0) \\
55(10.6) \\
51(9.4)\end{array}$ & $\begin{array}{l}47(15.6) \\
51(11.3) \\
50(13.0)\end{array}$ & $\begin{array}{l}45(13.1) \\
53(11.0) \\
48(11.1)\end{array}$ & $\begin{array}{l}47(18.3) \\
51(13.3) \\
49(13.9)\end{array}$ & $\begin{array}{l}44(13.3) \\
50(10.3) \\
48(11.2)\end{array}$ & $\begin{array}{l}4.5(7.8) \\
4.2(6.8) \\
2.2(4.9)\end{array}$ & $\begin{array}{r}0.0(4.0) \\
-1.2(4.0) \\
-3.2(3.7)\end{array}$ & $\begin{array}{l}2.0(8.1) \\
4.3(5.4) \\
3.0(5.1)\end{array}$ & $\begin{array}{r}-4.6(6.5) \\
4.3(5.4) \\
-2.8(4.7)\end{array}$ & $\begin{array}{c}20.1^{*}(1.1-40.4) \\
5.5^{*}(1.6-9.4) \\
7.3^{*}(2.7-11.9)\end{array}$ & $\begin{array}{l}4.7(-1.0 \text { to } 10.1) \\
6.6^{*}(0.1-13.1) \\
5.9^{*}(1.5-10.2)\end{array}$ \\
\hline
\end{tabular}

Abbreviations: $\mathrm{Cl}$, confidence interval; Con, control group (placebo therapy); Exp, experimental group (bronchodilator therapy); FEV1, forced expiratory volume in $1 \mathrm{~s}$; FVC, forced vital capacity; PEF, peak expiratory flow rate.

*significant $P<0.0$. 
was imprecision around this estimate as reflected in the $95 \%$ confidence interval. The lower end of the $95 \%$ confidence interval was $3 \%$. An effect this small may not be therapeutic. However, the upper end of the $95 \%$ confidence interval was $12 \%$. An effect this large may be therapeutic. The precision of all the estimates was reasonable despite the small sample size. This reflects the strength of cross-over trials for studies of this kind. Cross-over trials are more efficient and require fewer participants than parallel trials because participants act as their own controls. The research question explored in this trial was very conducive to a cross-over trial because the effects of bronchodilator therapy are short-lived minimising any potential for carry-over between the two phases of the study.

A limitation of this study is its external validity. Only a small sample of people from one spinal injury unit in Sydney was included. In addition, despite screening 118 patients admitted with recent tetraplegia, only 11 met the inclusion criteria (see Figure 1). The main reason for exclusion was a motor incomplete lesion. This exclusion criterion was used because we were primarily interested in understanding the effects of bronchodilator therapy in patients with minimal supraspinal control of the sympathetic nervous system. The effects of bronchodilator therapy in people with motor incomplete lesions requires further investigation. The next step would be to recruit a larger sample and compare regular administration of bronchodilator therapy over a sustained period of time with placebo in people with recently acquired tetraplegia and concurrent respiratory complications. It would be helpful to include outcome measures which reflect variables such as length of hospital stay, quality of life and serious respiratory complications. These types of outcome measures were not used in this study because we firstly wanted to establish the effect of bronchodilator therapy on surrogate outcomes such as FEV1, FVC and PEF. Without an effect on these outcomes, there is no obvious plausible way that bronchodilator therapy can affect more global health measures. Future studies may also want to examine the possible preventative effects of bronchodilator therapy on the development of respiratory complications. However, this type of trial will present a challenge to trialists.

\section{CONCLUSIONS}

This study demonstrates that bronchodilator therapy has short-term effects on FEV1, FVC and PEF in people with recently acquired tetraplegia. Further studies need to determine the therapeutic benefits of bronchodilator therapy provided regularly over an extended period of time and to people with concurrent respiratory complications.

\section{DATA ARCHIVING}

There were no data to deposit.

\section{CONFLICT OF INTEREST}

The authors declare no conflict of interest.

\section{ACKNOWLEDGEMENTS}

We acknowledge the assistance and co-operation of all the participants and Alex Avis, Jade Steadman, Jillian Eyles, Kirsty Saunders, Lydia Chen and Jayne Stanton who prepared the puffers and helped to provide the interventions. We also acknowledge the statistical advice received from Poppy Montgomery and Jonathon Sargent.

1 Winslow C, Rozovsky J. Effect of spinal cord injury on the respiratory system. Am J Phys Med Rehabil 2003; 82: 803-814.

2 Cosortium for Spinal Cord M. Respiratory management following spinal cord injury: a clinical practice guideline for health-care professionals. J Spinal Cord Med 2005; 28 : 259-293.

3 Almenoff PL, Alexander LR, Spungen AM, Lesser MD, Bauman WA. Bronchodilatory effects of ipratropium bromide in patients with tetraplegia. Paraplegia 1995; 33: 274-277.

4 DeLuca RV, Grimm DR, Lesser M, Bauman WA, Almenoff PL. Effects of a beta2-agonist on airway hyperreactivity in subjects with cervical spinal cord injury. Chest 1999; 115: 1533-1538.

5 Dicpinigaitis PV, Spungen AM, Bauman WA, Absgarten A, Almenoff PL. Bronchial hyperresponsiveness after cervical spinal cord injury. Chest 1994; 105: 1073-1076.

6 Fein ED, Grimm DR, Lesser M, Bauman WA, Almenoff PL. The effects of ipratropium bromide on histamine-induced bronchoconstriction in subjects with cervical spinal cord injury. J Asthma 1998; 35: 49-55.

7 Grimm DR, Schilero GJ, Spungen AM, Bauman WA, Lesser M. Salmeterol improves pulmonary function in persons with tetraplegia. Lung 2006; 184: 335-339.

8 Mateus SR, Beraldo PS, Horan TA. Cholinergic bronchomotor tone and airway caliber in tetraplegic patients. Spinal Cord 2006; 44: 269-274.

9 Kelley A, Garshick E, Gross ER, Lieberman SL, Tun CG, Brown R. Spirometry testing standards in spinal cord injury. Chest 2003; 123: 725-730.

10 Canning BJ. Reflex regulation of airway smooth muscle tone. J Appl Physiol 2006; 101: 971-985.

11 Baroffio M, Barisione G, Crimi E, Brusasco V. Noninflammatory mechanisms of airway hyper-responsiveness in bronchial asthma: an overview. Ther Adv Respir Dis 2009; 3 . 163-174.

12 Cockcroft DW, Davis BE. Mechanisms of airway hyperresponsiveness. J Allergy Clin Immunol 2006; 118: 551-559.

13 Meinero M, Coletta G, Dutto L, Milanese M, Nova G, Sciolla A et al. Mechanical response to methacholine and deep inspiration in supine men. J Appl Physiol 2007; 102: 269-275.

14 Torchio R, Gulotta C, Ciacco C, Perboni A, Guglielmo M, Crosa F et al. Effects of chest wall strapping on mechanical response to methacholine in humans. J App/ Physiol 2006; 101: 430-438.

15 Garstang SV, Miller-Smith SA. Autonomic nervous system dysfunction after spinal cord injury. Phys Med Rehabil Clin N Am 2007; 18: 275-296.

16 Warren J. The adrenal medulla and the airway. Br J Dis Chest 1986; 80: 1-6. 